\title{
Sur la densité de certains ensembles de multiples, 2
}

\author{
par
}

\section{A. RAOUJ (Marrakech)}

1. Introduction et énoncé des résultats. Considérons pour $n$ entier $\geq 1$ et $\lambda$ réel $\geq 0$, l'ensemble des multiples $\mathcal{B}_{\lambda}(n)$ de la suite

$$
\left.\left.\mathcal{D}_{\lambda}(n):=\bigcup_{d \mid n}\right] d, d \exp (\log n)^{-\lambda}\right] \cap \mathbb{N} .
$$

Désignons par $Q$ la fonction définie par $Q(\alpha):=\alpha \log \alpha-\alpha+1(\alpha>0)$.

Le but du présent travail est de montrer le résultat suivant.

THÉORÈme 1. On a

$$
\mathbf{d} \mathcal{B}_{\lambda}(n)=(\log n)^{-F(\lambda)+o(1)} \quad p . p .
$$

où l'on a posé

$$
F(\lambda)= \begin{cases}0 & \text { si } 0 \leq \lambda \leq \lambda^{*}=\log 4-1 \\ Q(\beta) & \text { si } \lambda^{*}<\lambda \leq \lambda^{* *}=\log 8-1 \\ & \text { avec } \beta=(1+\lambda) / \log 2-1 \\ \lambda-\log 2 & \text { si } \lambda^{* *}<\lambda\end{cases}
$$

Remarques. (i) La fonction $F$ est dérivable sur $[0, \infty[$.

(ii) Pour $\lambda \leq \lambda^{*}$ on a en fait $\mathbf{d} \mathcal{B}_{\lambda}(n)=1+o(1)$ p.p. [R93].

Interprétation probabiliste des valeurs critiques $\lambda^{*}$ et $\lambda^{* *}$. Considérons, pour $x$ entier, l'espace probabilisé $\Omega_{x}=\{1,2, \ldots, x\}$ muni de la loi uniforme $\nu_{x}$ et la suite $\left(\xi_{d}\right)_{d \geq 1}$ de variables aléatoires définies par

$$
\xi_{d}(n):= \begin{cases}1 & \text { si } d \mid n \\ 0 & \text { sinon. }\end{cases}
$$

Notant $H(x, y, z)$ le nombre des entiers $n \leq x$ ayant au moins un diviseur $d$ dans le sous-intervalle $] y, z]$ de $] 0, x]$, on a

$$
\frac{1}{x} H(x, y, z)=\frac{1}{x} \sum_{n \leq x} \max _{y<d \leq z} \xi_{d}(n)=\operatorname{Prob}\left(\max _{y<d \leq z} \xi_{d}=1\right) .
$$


Intuitivement, plus l'intervalle $] y, z]$ est petit plus les $\xi_{d}$ se rapprochent de l'indépendance. Cette dernière hypothèse implique

$$
\operatorname{Prob}\left(\max _{y<d \leq z} \xi_{d}=1\right)=1-\operatorname{Prob}\left(\max _{y<d \leq z} \xi_{d}=0\right) \approx 1-\prod_{y<d \leq z}\left(1-\frac{1}{x}\left[\frac{x}{d}\right]\right) .
$$

Il s'ensuit

$$
\frac{1}{x} H(x, y, z) \approx \frac{z-y}{y} .
$$

Remarquons en outre que l'inégalité de Behrend [B48] montre qu'on a toujours la majoration

$$
\frac{1}{x} H(x, y, z) \leq(1+\varepsilon(y)) \frac{z-y}{y} \quad\left(\lim _{y \rightarrow \infty} \varepsilon(y)=0\right) .
$$

Posons $z=y\left(1+(\log y)^{-\lambda}\right)$. Le théorème 21 de [HT88] montre que l'approximation (1.2) est effectivement valable si, et seulement si, $\lambda>\log 4-1$, ce qui représente donc le seuil d'indépendance pour ce problème.

Par ailleurs, désignons par $H\left(x, \mathcal{D}_{\lambda}(n)\right)$ le nombre des entiers $m \leq x$ $\left(x \geq x_{0}(\lambda, n)\right)$ ayant au moins un diviseur dans $\mathcal{D}_{\lambda}(n)$; l'hypothèse d'indépendance des $\xi_{t}\left(t \in \mathcal{D}_{\lambda}(n)\right)$ implique

$$
\frac{1}{x} H\left(x, \mathcal{D}_{\lambda}(n)\right)=\operatorname{Prob}\left(\max _{t \in \mathcal{D}_{\lambda}(n)} \xi_{t}=1\right)=(\log n)^{\log 2-\lambda+o(1)} \quad \text { p.p. }
$$

car en utilisant la majoration (voir [HT88], Theorem 56)

$$
\Delta(n):=\max _{z} \operatorname{card}\{d: d \mid n, z<d \leq e z\} \leq\left(\log _{2} n\right)^{1+o(1)} \quad \text { p.p. }
$$

on montre facilement que

$$
\sum_{m \in \mathcal{D}_{\lambda}(n)} 1 / m=(\log n)^{\log 2-\lambda+o(1)} \quad \text { p.p. }
$$

Ainsi, la valeur $\lambda^{* *}=\log 8-1$ de $\lambda$ du théorème 1 peut être interprétée comme le seuil d'indépendance des $\xi_{t}\left(t \in \mathcal{D}_{\lambda}(n)\right)$.

De plus, en écrivant

$$
\max _{t \in \mathcal{D}_{\lambda}(n)} \xi_{t}=\max _{d \mid n} \max _{d<t \leq\left(1+(\log n)^{-\lambda}\right) d} \xi_{t}=\max _{d \mid n} \xi_{d}^{*} \text { disons, }
$$

on voit que, pour $\lambda<\lambda^{* *}$, les $\xi_{d}^{*}$ ne sont plus indépendants, alors que, pour chaque $d \mid n, \lambda^{*}$ représente, au vu du théorème 21 de [HT88], le seuil d'indépendance des $\xi_{t}$, pour $d<t \leq\left(1+(\log d)^{-\lambda}\right) d$.

Commençons par établir les lemmes utiles.

2. Lemmes. Le lemme suivant, dû à Halberstam et Richert [HR79] et généralisant un résultat de Hall, nous sera très utile. 
LEMME 1 (voir [T90]). Si $f$ est une fonction multiplicative, positive et à laquelle on peut associer un couple $\left(\lambda_{1}, \lambda_{2}\right)$ de $\mathbb{R}^{+} \times[0,2[$ tel que pour tout nombre premier $p$ et pour tout entier $j>0$ on ait

$$
f\left(p^{j}\right) \leq \lambda_{1} \lambda_{2}^{j-1}
$$

alors, pour tout réel $x \geq 2$,

$$
\sum_{n \leq x} f(n) \leq 67\left(1+9 \lambda_{1}+\lambda_{1} \lambda_{2}\left(2-\lambda_{2}\right)^{-2}\right) x \prod_{p \leq x}\left(1-p^{-1}\right) \sum_{j \geq 0} f\left(p^{j}\right) p^{-j} .
$$

Le résultat suivant dû à Shiu [S80] nous sera utile. On considère la classe $\mathcal{F}$ des fonctions multiplicatives vérifiant les deux propriétés suivantes :

$\left(\mathcal{F}_{1}\right)$ il existe une constante $A_{1}>0$ telle que pour tout nombre premier $p$ et pour tout entier $\nu>0$,

$$
0 \leq f\left(p^{\nu}\right) \leq A_{1}^{\nu},
$$

$\left(\mathcal{F}_{2}\right)$ pour tout $\varepsilon>0$, il existe une constante $A_{2}=A_{2}(\varepsilon)$ telle que pour tout entier $n>0$,

$$
f(n) \leq A_{2} n^{\varepsilon} .
$$

Lemme 2. Soient $f \in \mathcal{F}, 0<\alpha<1 / 2$ et $x^{\alpha}<y \leq x$. Alors, uniformément pour $y$ et $x$, on a

$$
\sum_{x-y<n \leq x} f(n) \ll_{f, \alpha} y(\log x)^{-1} \exp \left(\sum_{p \leq x} f(p) / p\right) .
$$

On désigne exclusivement par la lettre $p$ un nombre premier. $\omega(n)$ est le nombre de facteurs premiers de $n$ et $\Omega(n)$ est le nombre de facteurs premiers de $n$, comptés avec leurs ordres de multiplicités.

Le lemme suivant est une conséquence du lemme 2.

Lemme 3. Uniformément pour $0<\alpha \leq 1,0<y<z$ et $0<m \leq z-y$, on $a$

$$
\sum_{y / m<t \leq z / m} \frac{\alpha^{\Omega(t)}}{t} \ll \frac{z-y}{y}\left(\log \frac{y}{z-y}\right)^{1-\alpha}\left(\log \frac{z}{m}\right)^{\alpha-1} .
$$

Démonstration. Distinguons deux cas, selon que $y / m>(y /(z-y))^{3 / 2}$ ou non. Dans le premier cas nous avons $(z-y) / m>(y / m)^{1 / 3}$ et donc $(2.3)$ résulte du lemme 2. Dans le second cas, la majoration triviale

$$
\sum_{y / m<t \leq z / m} 1 / t \ll \log (z / y)+m / y \ll(z-y) / y
$$

suffit. 
On pose

$$
\Omega(n, t):=\sum_{\substack{j \\ p^{j} \mid n, j>0 \\ p \leq t}} 1, \quad \omega(n, t):=\sum_{\substack{p \mid n \\ p \leq t}} 1
$$

$P^{+}(n)$ le plus grand facteur premier de $n$ et $\mu(n):=(-1)^{\omega(n)}$ si $n$ est sans facteur carré, sinon $\mu(n):=0$.

Le lemme 3 sera appliqué pour démontrer le résultat suivant.

Lemme 4. Soient $0<y \leq 1$ et $\lambda>0$. Pour $n$ assez grand, posons $\varepsilon=\varepsilon(n)=\log _{3} n / \sqrt{\log _{2} n}, T(n)=\exp (\log n)^{1-\varepsilon}$ et $\eta=(\log n)^{-\lambda}$. Alors $i l$ existe une fonction $X_{0}(n)$ telle que pour $X \geq X_{0}(n)$ on ait

$$
\sum_{\substack{d \mid n \\ P^{+}(d)>T(n)}} \mu(d)^{2} \sum_{m \leq X} y^{\Omega(m, 3 n)} \sum_{\substack{t, t^{\prime} \mid m \\ 0<t, t^{\prime}<(1+\eta) d}} 1 \leq X(\log n)^{E} \quad p . p .
$$

où l'on a posé $E=4 y-3-2 \lambda+\log 2+3 \varepsilon$.

Démonstration. Notons $S=S(X, n)$ la somme ci-dessus. Si $t$ et $t^{\prime}$ sont comptés dans la somme intérieure de $S$ alors en posant

$$
t_{0}=\left(t, t^{\prime}\right), \quad t=s t_{0}, \quad t^{\prime}=s^{\prime} t_{0},
$$

on a $\left[t, t^{\prime}\right]=t_{0} s s^{\prime}, d / t_{0}<s<s^{\prime} \leq(1+\eta) d / t_{0}$ et $t_{0} \leq \eta d$. Donc

$$
\begin{aligned}
S \leq & \sum_{\substack{d \mid n \\
P^{+}(d)>T(n)}} \mu(d)^{2} \sum_{t_{0} \leq(1+\eta) d} y^{\Omega\left(t_{0}\right)} \sum_{d / t_{0}<s \leq(1+\eta) d / t_{0}} y^{\Omega(s)} \\
& \times \sum_{d / t_{0}<s^{\prime} \leq(1+\eta) d / t_{0}} y^{\Omega\left(s^{\prime}\right)} \sum_{m \leq X /\left(t_{0} s s^{\prime}\right)} y^{\Omega(m, 3 n)} .
\end{aligned}
$$

Utilisant les lemmes 1 et 3 on peut écrire pour $X_{0}(n) \geq n^{3}$,

$$
\begin{gathered}
\sum_{m \leq X /\left(t_{0} s s^{\prime}\right)} y^{\Omega(m, 3 n)} \ll\left(X /\left(t_{0} s s^{\prime}\right)\right)(\log n)^{y-1}, \\
\sum_{d / t_{0}<s \leq(1+\eta) d / t_{0}} y^{\Omega(s)} / s \ll \eta(\log (1 / \eta))^{1-y}\left(\log \left(d / t_{0}\right)\right)^{y-1},
\end{gathered}
$$

d'où

$$
\begin{aligned}
S \ll & X(\log n)^{y-1-2 \lambda}\left(\lambda \log _{2} n\right)^{2-2 y} \\
& \times \sum_{\substack{d \mid n \\
P^{+}(d)>T(n)}} \mu(d)^{2} \sum_{t_{0} \leq \eta d}\left(\log \left(d / t_{0}\right)\right)^{2 y-2} t_{0}^{-1} y^{\Omega\left(t_{0}\right)} .
\end{aligned}
$$

Désignons par $S^{\prime}$ la dernière somme en $t_{0}$. Par intégration par parties nous 
avons

$$
\begin{aligned}
& S^{\prime} \ll(\log (1 / \eta))^{2 y-2}(\log d)^{y-1}+(\log d)^{2 y-2} \int_{1}^{\sqrt{d}}(\log t)^{y-1} t^{-1} d t \\
& \quad+(\log d)^{y-1} \int_{\sqrt{d}}^{\eta d}(\log (d / t))^{2 y-2} t^{-1} d t \\
& \leq(\log d)^{y-1+(2 y-1)^{+}}\left(\log _{2} n\right)^{2} \quad\left(n \geq n_{0}(y, \lambda)\right) .
\end{aligned}
$$

On a donc

$$
S \ll_{y, \lambda} X(\log n)^{y-1-2 \lambda}\left(\log _{2} n\right)^{4-2 y} \sum_{\substack{d \mid n \\ P^{+}(d)>T(n)}} \mu(d)^{2}(\log d)^{3 y-2} .
$$

La dernière somme en $d$ est majorée par

$$
(\log n)^{3 y-2+(2-3 y)^{+} \varepsilon} 2^{\omega(n)} \leq(\log n)^{3 y-2+\log 2+\varepsilon+(2-3 y)^{+} \varepsilon} \quad \text { p.p. }
$$

On obtient ainsi $S \leq X(\log n)^{4 y-3-2 \lambda+\log 2+3 \varepsilon}$ p.p.

Lemme 5. Pour $0.33 \leq \alpha<1$, on $a$

$$
\inf _{x \geq 1} \int_{0}^{x} t^{-\alpha} \cos t d t \geq 9 \cdot 10^{-3}
$$

Démonstration. Pour $0<\alpha<1$ et $k \in \mathbb{N}$, notons

$$
F_{\alpha}(x):=\int_{0}^{x} t^{-\alpha} \cos t d t, \quad m_{k}:=\min _{\pi / 2+2 k \pi \leq x \leq \pi / 2+(2 k+2) \pi} F_{\alpha}(x) .
$$

On a

$$
\begin{aligned}
m_{k} & =F_{\alpha}(\pi / 2+(2 k+1) \pi) \\
& =m_{k-1}+\int_{3 \pi / 2}^{7 \pi / 2}(t+2(k-1) \pi)^{-\alpha} \cos t d t \\
& =m_{k-1}+\int_{3 \pi / 2}^{5 \pi / 2}\left((t+2(k-1) \pi)^{-\alpha}-(t+(2 k-1) \pi)^{-\alpha}\right) \cos t d t .
\end{aligned}
$$

En particulier, $m_{k}>m_{k-1}(k \geq 1)$.

Comme $F_{\alpha}$ est croissante sur [1, $\left.\pi / 2\right]$, on peut écrire

$$
\inf _{x \geq 1} F_{\alpha}(x)=\min \left(F_{\alpha}(3 \pi / 2), F_{\alpha}(1)\right) .
$$

En utilisant l'estimation

$$
\cos t \geq 1-t^{2} / 2+t^{4} / 4 !-t^{6} / 6 !+t^{8} / 8 !-t^{10} / 10 ! \quad(t \geq 0),
$$


on peut écrire

$$
F_{\alpha}(3 \pi / 2) \geq(3 \pi / 2)^{1-\alpha} h(\alpha)
$$

où l'on a posé

$$
\begin{aligned}
h(\alpha)= & \frac{1}{1-\alpha}-\frac{1}{2(3-\alpha)}(3 \pi / 2)^{2}+\frac{1}{4 !(5-\alpha)}(3 \pi / 2)^{4} \\
& -\frac{1}{6 !(7-\alpha)}(3 \pi / 2)^{6}+\frac{1}{8 !(9-\alpha)}(3 \pi / 2)^{8}-\frac{1}{10 !(11-\alpha)}(3 \pi / 2)^{10} .
\end{aligned}
$$

En effectuant une vérification numérique il vient $h(0.33)=0.0097 \ldots>0$. Le lemme 5 sera donc prouvé si on montre que $h$ est croissante sur $[0.33,1[$. Posons

$$
\begin{aligned}
& h_{1}(\alpha):=\frac{1}{1-\alpha}-\frac{1}{2(3-\alpha)}(3 \pi / 2)^{2}, \\
& h_{2}(\alpha):=\frac{1}{5-\alpha}-\frac{1}{30(7-\alpha)}(3 \pi / 2)^{2}, \\
& h_{3}(\alpha):=\frac{1}{9-\alpha}-\frac{1}{90(11-\alpha)}(3 \pi / 2)^{2},
\end{aligned}
$$

de sorte que $h(\alpha)=\sum_{i=1}^{3} c_{i} h_{i}(\alpha)\left(c_{i} \geq 0\right)$. On vérifie aisément que $h_{i}^{\prime}(\alpha) \geq 0$ $(i=1,2,3)$ pour $1 / 4 \leq \alpha<1$. Cela montre que $h$ est croissante sur $[1 / 4,1[$.

Le lemme suivant est une conséquence immédiate du lemme 5.

Lemme 6 . Pour $0<\varrho \leq 0.67$ et $u \in \mathbb{R}$, on $a$

$$
(1+|u|)^{-\varrho} \leq 100 \int_{-1}^{1}|t|^{\varrho-1} e^{i t u} d t .
$$

Démonstration. On a

$$
\int_{-1}^{1}|t|^{\varrho-1} e^{i u t} d t=2 \int_{0}^{1} t^{\varrho-1} \cos (u t) d t=2|u|^{-\varrho} \int_{0}^{|u|} t^{\varrho-1} \cos t d t .
$$

Distinguons le cas où $|u| \leq 1$ et $|u|>1$. Dans le premier cas nous avons

$$
2|u|^{-\varrho} \int_{0}^{|u|} t^{\varrho-1} \cos t d t \geq 2 \varrho^{-1} \cos 1 \geq 1 \geq(1+|u|)^{-\varrho} .
$$

Dans le second cas on a, d'après le lemme 5 ,

$$
2|u|^{-\varrho} \int_{0}^{|u|} t^{\varrho-1} \cos t d t \geq 10^{-2}|u|^{-\varrho} \geq 10^{-2}(1+|u|)^{-\varrho} .
$$

On pose $n_{k}:=\prod_{p \mid n, p \leq \operatorname{expe} e^{k}} p$.

Nous avons besoin du lemme 6 pour établir le résultat suivant. 
Lemme 7. Soient $\varepsilon>0$ suffisamment petit, $0<\varrho \leq 0.67, \varrho / \log 2<$ $\alpha<1, \delta>1$ et $\log (1 / \varepsilon)<k \leq \log _{2} x$. On $a$

$$
\sum_{d, d^{\prime} \mid n_{k}}\left(1+\left|\log \left(d^{\prime} / d\right)\right|\right)^{-\varrho} \leq \varrho^{-1} \varepsilon^{-1} e^{(\delta \log 4-\varrho) k}
$$

pour tous les entiers $n \leq x$ sauf

$$
\ll x\left(Q(\alpha)^{-1} \varepsilon^{Q(\alpha)}+e^{-Q(\delta) k}+(\alpha \log 2-\varrho)^{-1} \varepsilon^{\alpha \log 2-\varrho}\right) .
$$

Démonstration. D'après le lemme 6 on a

$$
\begin{aligned}
\sum_{d, d^{\prime} \mid n_{k}}\left(1+\left|\log \frac{d^{\prime}}{d}\right|\right)^{-\varrho} & \leq 100 \int_{-1}^{1} \sum_{d, d^{\prime} \mid n_{k}} e^{i \theta \log \left(d^{\prime} / d\right)} \frac{d \theta}{|\theta|^{1-\varrho}} \\
& \leq 200 \int_{0}^{1}\left|\tau^{*}\left(n_{k}, \theta\right)\right|^{2} \frac{d \theta}{\theta^{1-\varrho}}
\end{aligned}
$$

où

$$
\tau^{*}\left(n_{k}, \theta\right)=\sum_{d \mid n_{k}} \mu(d)^{2} d^{i \theta} .
$$

Pour $k$ fixé, on sait (voir le lemme 5.1 de [R93]) que $\omega\left(n_{k}\right)<\delta k$ pour tous les entiers $n \leq x$ à l'exception d'au plus $O\left(x e^{-Q(\delta) k}\right)$. Pour ces entiers $n$ non exceptionnels on a

$$
\int_{0}^{1 /\left(\varepsilon e^{k}\right)}\left|\tau^{*}\left(n_{k}, \theta\right)\right|^{2} \frac{d \theta}{\theta^{1-\varrho}} \leq \tau\left(n_{k}\right)^{2} \int_{0}^{1 /\left(\varepsilon e^{k}\right)} \frac{d \theta}{\theta^{1-\varrho}}=4^{\omega\left(n_{k}\right)} \varrho^{-1} \varepsilon^{-\varrho} e^{-k \varrho},
$$

ce qui entraîne

$$
\int_{0}^{1 /\left(\varepsilon e^{k}\right)}\left|\tau^{*}\left(n_{k}, \theta\right)\right|^{2} \frac{d \theta}{\theta^{1-\varrho}} \leq \varrho^{-1} e^{(\delta \log 4-\varrho) k} \varepsilon^{-\varrho}
$$

pour tous les entiers $n \leq x$ sauf $\ll x e^{-Q(\delta) k}$. Par ailleurs, posant $\omega_{\theta}(n):=\operatorname{card}\{p: p \mid n, p \leq \exp (1 / \theta)\}$ et $\mathcal{R}(n, \theta):=\left|\tau^{*}(n, \theta)\right|^{2} / 2^{\omega(n)+\omega_{\theta}(n)}$ on peut écrire

$$
\int_{1 /\left(\varepsilon e^{k}\right)}^{1}\left|\tau^{*}\left(n_{k}, \theta\right)\right|^{2} \frac{d \theta}{\theta^{1-\varrho}}=4^{\omega\left(n_{k}\right)} \int_{1 /\left(\varepsilon e^{k}\right)}^{1} \frac{\mathcal{R}\left(n_{k}, \theta\right)}{2^{\omega\left(n_{k}\right)-\omega_{\theta}\left(n_{k}\right)}} \cdot \frac{d \theta}{\theta^{1-\varrho}} .
$$

Répartissons alors les entiers $n \leq x$ pour lesquels cette quantité excède $4^{\delta k} e^{-\varrho k}$ en trois classes $\mathcal{C}_{1}, \mathcal{C}_{2}, \mathcal{C}_{3}$ définies par les conditions

$$
\begin{gathered}
\omega\left(n_{k}\right)>\delta k, \\
\min _{1 /\left(\varepsilon e^{k}\right)<\theta \leq 1} \frac{\omega\left(n_{k}\right)-\omega_{\theta}\left(n_{k}\right)}{\log \left(\theta e^{k}\right)}<\alpha,
\end{gathered}
$$


$\left(\mathcal{C}_{3}\right)$

$$
\omega\left(n_{k}\right) \leq \delta k \quad \text { et } \quad \min _{1 /\left(\varepsilon e^{k}\right)<\theta \leq 1} \frac{\omega\left(n_{k}\right)-\omega_{\theta}\left(n_{k}\right)}{\log \left(\theta e^{k}\right)} \geq \alpha .
$$

Utilisant le lemme 51.2 de [HT88], on a

$$
\left|\mathcal{C}_{2}\right| \ll x Q(\alpha)^{-1} \varepsilon^{Q(\alpha)} .
$$

En outre,

$$
\begin{aligned}
\left|\mathcal{C}_{3}\right| & \leq 4^{-\delta k} e^{\varrho k} \sum_{n \leq x} 4^{\omega\left(n_{k}\right)} \int_{1 /\left(\varepsilon e^{k}\right)}^{1} \frac{\mathcal{R}\left(n_{k}, \theta\right)}{2^{\omega\left(n_{k}\right)-\omega_{\theta}\left(n_{k}\right)}} \cdot \frac{d \theta}{\theta^{1-\varrho}} \\
& \leq e^{\varrho k} \sum_{n \leq x} \int_{1 /\left(\varepsilon e^{k}\right)}^{1} \frac{\mathcal{R}\left(n_{k}, \theta\right)}{\left(\theta e^{k}\right)^{\alpha \log 2}} \cdot \frac{d \theta}{\theta^{1-\varrho}} .
\end{aligned}
$$

Et puisque pour $k \geq 0$ et $\theta$ réel $>0$, on a

$$
\sum_{n \leq x} \mathcal{R}\left(n_{k}, \theta\right) \ll x
$$

(voir lemme 6.1 de [R93]), on obtient donc

$$
\left|\mathcal{C}_{3}\right| \ll x(\alpha \log 2-\varrho)^{-1} \varepsilon^{\alpha \log 2-\varrho} .
$$

Cela achève la démonstration du lemme 7 .

Le lemme suivant nous sera utile :

Lemme 8 (voir [T90], Théorème II.6.4). Soit $A>0$. Il existe des constantes positives $c_{1}=c_{1}(A)$ et $c_{2}=c_{2}(A)$ telles que uniformément pour $x \geq 3$ et $1 \leq k \leq A \log _{2} x$, on ait

$$
\sum_{\substack{n \leq x \\ \omega(n)=k}} 1=\frac{x}{\log x} \cdot \frac{\left(\log _{2} x\right)^{k-1}}{(k-1) !}\left\{\lambda\left(\frac{k-1}{\log _{2} x}\right)+O\left(\frac{k}{\left(\log _{2} x\right)^{2}}\right)\right\}
$$

avec

$$
\lambda(z):=\frac{1}{\Gamma(z+1)} \prod_{p}\left(1+\frac{z}{p-1}\right)\left(1-\frac{1}{p}\right)^{z} .
$$

Le lemme suivant est un résultat classique du crible. On désigne par $\Psi(x, y)$ le nombre des entiers $n \leq x$ tels que $P^{+}(n) \leq y$.

Lemme 9 (voir [T90], p. 396). Pour $x \geq y \geq 2$, on a

$$
\Psi(x, y) \ll x \exp (-\log x /(2 \log y)) .
$$

On désigne par $\Phi(x, z)$ le nombre des entiers $n \leq x$ tels que $P^{-}(n)>z$.

Lemme 10 (voir [HT88], p. 11). Pour $x \geq 2 z \geq 4$, on a

$$
\Phi(x, z) \asymp x / \log z .
$$


Lemme 11 (voir [HR66], p. 147). Soient $n \geq 1$ et $\left(x_{1}, \ldots, x_{n}\right) \in \mathbb{R}_{+}^{n}$. Posant pour chaque entier $k$ de $\{1, \ldots, n\}$,

$$
S_{k}:=\sum_{\substack{I \subset\{1, \ldots, n\} \\|I|=k}} \prod_{i \in I} x_{i},
$$

on $a$

$$
S_{k} \geq\left\{1-\left(\begin{array}{l}
k \\
2
\end{array}\right) \frac{\sum_{i=1}^{n} x_{i}^{2}}{S_{1}^{2}}\right\} \frac{S_{1}^{k}}{k !} .
$$

Le résultat suivant fournit une condition suffisante sur $\lambda$ pour que les intervalles $\left.] d,\left(1+(\log n)^{-\lambda}\right) d\right], d \mid n$, soient deux à deux disjoints.

Lemme 12 (voir théorème 54 de [HT88]). Posant $E(n):=\min \left\{\log \left(d^{\prime} / d\right):\right.$ $\left.d, d^{\prime} \mid n, d<d^{\prime}\right\}$, on a

$$
E(n)=(\log n)^{1-\log 3} \exp \left\{O\left(\sqrt{\log _{2} n \cdot \log _{3} n}\right)\right\} \quad p \cdot p .
$$

Dans le paragraphe suivant, nous regroupons les notations et les conventions que nous allons introduire dans la démonstration du théorème 2 .

Notations et conventions. Posons

$$
\begin{aligned}
& \beta=\beta_{\lambda}:= \begin{cases}\frac{1+\lambda}{\log 2}-1 & \text { si } \log 4-1<\lambda \leq \log 8-1, \\
2 & \text { si } \lambda>\log 8-1,\end{cases} \\
& F=F(\lambda):= \begin{cases}Q(\beta) & \text { si } \log 4-1<\lambda \leq \log 8-1, \\
\lambda-\log 2 & \text { si } \lambda>\log 8-1,\end{cases} \\
& K=K(n, \beta):=\left[\frac{\beta}{2} \log _{2} n\right], \\
& \mathcal{S}=\mathcal{S}(X ; n, \beta):=\left\{m \leq X: \Omega(m, 3 n) \leq \beta \log _{2} n\right\}, \\
& \varepsilon=\varepsilon(n):=\frac{\log _{3} n}{\sqrt{\log _{2} n}}, \\
& T=T(n):=\exp \left\{(\log n)^{1-\varepsilon(n)}\right\}, \\
& \eta:=(\log n)^{-\lambda}, \\
& \left.\left.\mathcal{D}_{n}^{*}:=\bigcup_{\substack{d \mid n, \mu(d)^{2}=1 \\
P^{+}(d)>T(n)}}\right] d,(1+\eta) d\right], \\
& \left.\left.\mathcal{D}_{n}:=\bigcup_{d \mid n}\right] d,(1+\eta) d\right], \\
& \tau(m, \mathcal{A}):=\sum_{t \mid m, t \in \mathcal{A}} 1 \quad(\mathcal{A} \subset \mathbb{N}), \\
& B(X ; n, \lambda):=|\{m \leq X: \tau(m, \mathcal{D})>0\}| .
\end{aligned}
$$


3. La minoration de $\mathbf{d} \mathcal{B}_{\lambda}(n)$. Soient $n \geq 1$ et $X \geq X_{0}(n)$ choisi comme dans l'énoncé du lemme $4,0<y_{0}, y_{1} \leq 1$. Considérons parmi les entiers $m$ comptés dans $\mathcal{S}$ ceux qui s'écrivent sous la forme $m=a b$ avec

$$
a \in \mathcal{D}_{n}^{*}, \quad \omega(a) \leq K(n, \beta), \quad \omega(b, 3 n) \leq K(n, \beta) \quad \text { et } b \leq X / a .
$$

On a

$$
\sum_{m \in \mathcal{S}} \tau\left(m, \mathcal{D}_{n}^{*}\right) \geq \sum_{\substack{a \in \mathcal{D}_{n}^{*} \\ \omega(a) \leq K}} \sum_{\substack{b \leq X / a \\ \omega(b, 3 n) \leq K}} 1 .
$$

Faisant appel aux lemmes 10 et 11, on peut écrire

$$
\begin{aligned}
\sum_{\substack{b \leq X / a \\
\omega(b, 3 n) \leq K}} 1 \geq \sum_{\substack{b_{1} \\
P^{+}\left(b_{1}\right) \leq 3 n \\
\omega\left(b_{1}\right)=K}} \mu\left(b_{1}\right)^{2} \sum_{\substack{b_{2} \leq X /\left(a b_{1}\right) \\
P^{-}\left(b_{2}\right)>3 n}} 1 \\
\gg \frac{X}{a \log n} \sum_{\substack{b_{1} \\
P^{+}\left(b_{1}\right) \leq 3 n \\
\omega\left(b_{1}\right)=K}} \mu\left(b_{1}\right)^{2} / b_{1} \\
\gg \frac{X}{a \log n} \cdot \frac{1}{K !}\left(\log _{2} n+O(1)\right)^{K} .
\end{aligned}
$$

La formule de Stirling entraîne alors

$$
\sum_{\substack{b \leq X / a \\ \omega(b, 3 n) \leq K}} 1 \geq(X / a)(\log n)^{-Q(\beta / 2)+o(1)} .
$$

Il en découle

$$
\sum_{m \in \mathcal{S}} \tau\left(m, \mathcal{D}_{n}^{*}\right) \geq X(\log n)^{-Q(\beta / 2)+o(1)} \sum_{\substack{a \in \mathcal{D}^{*} \\ \omega(a) \leq K}} 1 / a .
$$

Puisque $\lambda>\log 3-1$, le lemme 12 montre que si $n$ est dans une suite convenable de densité 1 alors les intervalles $] d,(1+\eta) d], d \mid n$, sont deux à deux disjoints. Par conséquent,

$$
\begin{aligned}
& \sum_{m \in \mathcal{S}} \tau\left(m, \mathcal{D}^{*}\right) \\
& \quad \geq X(\log n)^{-Q(\beta / 2)+o(1)} \sum_{\substack{d \mid n \\
P^{+}(d)>T(n)}} \mu(d)^{2} \sum_{\substack{d<a \leq(1+\eta) d \\
\omega(a) \leq K}} 1 / a .
\end{aligned}
$$

Appliquant le lemme 8 on a pour chaque $d$ tel que $d>T(n)$,

$$
\sum_{\substack{d<a \leq(1+\eta) d \\ \omega(a)=K}} 1 / a \gg \eta(\log d)^{-1}\left(\log _{2} d\right)^{K-1}(K-1) !^{-1} .
$$


Il suit

$$
\begin{aligned}
\sum_{m \in \mathcal{S}} \tau\left(m, \mathcal{D}^{*}\right) \geq & X(\log n)^{-Q(\beta / 2)-\lambda+o(1)}(K-1) !^{-1} \\
& \times \sum_{\substack{d \mid n \\
P^{+}(d)>T(n)}}\left(\log _{2} d\right)^{K-1}(\log d)^{-1} \mu(d)^{2} \\
\geq & X(\log n)^{-Q(\beta / 2)-\lambda+o(1)}\left((1-\varepsilon) \log _{2} n\right)^{K-1} \\
& \times \frac{1}{(K-1) !} \sum_{\substack{d \mid n \\
P^{+}(d)>T(n)}} \mu(d)^{2} .
\end{aligned}
$$

D'après le lemme 9 , on a $P^{+}(n)>T(n)$ p.p. Cela implique que la somme en $d$ est supérieure ou égale à $(\log n)^{(1-\varepsilon) \log 2}$ p.p. Par ailleurs, la formule de Stirling montre que

$$
(1-\varepsilon)^{K-1}\left(\log _{2} n\right)^{K-1}(K-1) !^{-1} \geq e^{-2 \varepsilon K}(\log n)^{1-Q(\beta / 2)} \quad \text { p.p. }
$$

Il vient donc

$$
\sum_{m \in \mathcal{S}} \tau\left(m, \mathcal{D}^{*}\right) \geq X(\log n)^{-(2 Q(\beta / 2)+\lambda-\log 2)+o(1)} \quad \text { p.p. }
$$

Pour $\lambda>\log 4-1$ on a $2 Q(\beta / 2)+\lambda-\log 2=F(\lambda)$, d'où

$$
\inf _{X \geq X_{0}(n)}(1 / X) \sum_{m \in \mathcal{S}(X ; n, \beta)} \tau\left(m, \mathcal{D}_{n}^{*}\right) \geq(\log n)^{-F(\lambda)+o(1)} \quad \text { p.p. }
$$

Par ailleurs, pour minorer $\mathbf{d} \mathcal{B}_{\lambda}(n)$ on utilise l'inégalité de Cauchy-Schwarz :

$$
\mathbf{d} \mathcal{B}_{\lambda}(n) \geq \liminf _{X \rightarrow \infty}\left\{\sum_{m \in \mathcal{S}} \tau\left(m, \mathcal{D}_{n}^{*}\right)\right\}^{2} /\left\{X \sum_{m \in \mathcal{S}} \tau\left(m, \mathcal{D}_{n}^{*}\right)^{2}\right\} .
$$

On est donc conduit à majorer $\sum_{m \in \mathcal{S}} \tau\left(m, \mathcal{D}_{n}^{*}\right)^{2}$. On a

$$
\begin{aligned}
& \sum_{m \in \mathcal{S}} \tau\left(m, \mathcal{D}_{n}^{*}\right)^{2} \\
& \leq 2 \sum_{\substack{d \mid n \\
P^{+}(d)>T(n)}} \mu(d)^{2} \sum_{\substack{d^{\prime} \mid n, d^{\prime} \geq d \\
P^{+}\left(d^{\prime}\right)>T(n)}} \mu\left(d^{\prime}\right)^{2} \sum_{m \in \mathcal{S}} \sum_{\begin{array}{c}
t, t^{\prime} \mid m \\
d<t \leq(1+\eta) d \\
d^{\prime}<t^{\prime} \leq(1+\eta) d^{\prime}
\end{array}} 1 .
\end{aligned}
$$

Si $t$ et $t^{\prime}$ sont comptés dans cette dernière somme intérieure, alors en posant

$$
r=\left(t, t^{\prime}\right), \quad s=t / r, \quad s^{\prime}=t^{\prime} / r,
$$

on a

$$
\begin{gathered}
{\left[t, t^{\prime}\right]=r s s^{\prime},} \\
d / r<s \leq(1+\eta) d / r, \quad d^{\prime} / r<s \leq(1+\eta) d^{\prime} / r,
\end{gathered}
$$




$$
r \leq 1+\eta d \quad \text { si } s \neq 1 .
$$

Nous allons en fait décomposer la somme en question selon les cas

$$
s=s^{\prime}=1, \quad s=1 \text { et } s^{\prime}>1, \quad s>1 \text { et } s^{\prime}>1
$$

en remarquant que le cas $s>1$ et $s^{\prime}=1$ est impossible. Notons alors

$$
\begin{aligned}
S_{0}(X ; n, d):= & \sum_{\substack{m \leq X \\
\Omega(m, 3 n) \leq \beta \log 2 n}}\left(\sum_{\substack{t \mid m \\
d<t \leq(1+\eta) d}} 1\right)^{2}, \\
S_{1}\left(X ; n, d, d^{\prime}\right):= & (\log n)^{-\beta \log y_{1}} \sum_{\substack{d<r \leq(1+\eta) d \\
\Omega(r)}} y_{1}^{\Omega\left(s^{\prime}\right)} \sum_{m \leq X /\left(r s^{\prime}\right)} y_{1}^{\Omega(m, 3 n)}, \\
& \times \sum_{d^{\prime} / r<s^{\prime} \leq(1+\eta) d^{\prime} / r} y_{1}^{\Omega(r)} \sum_{m} y_{2}^{\Omega(s)} \\
S_{2}\left(X ; n, d, d^{\prime}\right):= & (\log n)^{-\beta \log y_{2}} \sum_{r \leq(1+\eta) d / 2} y_{2}^{\Omega(r)} \sum_{d / r<s \leq(1+\eta) d / r} y_{2}^{\Omega\left(s^{\prime}\right)} \sum_{m \leq X /\left(r s s^{\prime}\right)} y_{2}^{\Omega(m, 3 n)} .
\end{aligned}
$$

On a

$$
\begin{aligned}
& \sum_{m \in \mathcal{S}} \tau\left(m, \mathcal{D}^{*}\right)^{2} \\
& \leq \sum_{\substack{d \mid n \\
P^{+}(d)>T(n)}} \mu(d)^{2} S_{0}(X ; n, d) \\
& +2 \sum_{\substack{d\left|n, d^{\prime}\right| n \\
P^{+}(d)>T(n), P^{+}\left(d^{\prime}\right)>T(n) \\
d^{\prime}>d}} \mu(d)^{2} \mu\left(d^{\prime}\right)^{2}\left\{S_{1}\left(X ; n, d, d^{\prime}\right)+S_{2}\left(X ; n, d, d^{\prime}\right)\right\} .
\end{aligned}
$$

D'après les lemmes 1 et 3 on a

$$
\begin{aligned}
S_{2}\left(X ; n, d, d^{\prime}\right) \ll & X(\log n)^{y_{2}-1-\beta \log y_{2}} \sum_{r \leq(1+\eta) d / 2} y_{2}^{\Omega(r)} / r \\
& \times \sum_{d / r<s \leq(1+\eta) d / r} y_{2}^{\Omega(s)} / s \sum_{d^{\prime} / r<s^{\prime} \leq(1+\eta) d^{\prime} / r} y_{2}^{\Omega\left(s^{\prime}\right)} / s^{\prime} \\
\ll & X(\log n)^{y_{2}-1-\beta \log y_{2}-2 \lambda}(\log (1 / \eta))^{2-2 y_{2}} \\
& \times \sum_{r \leq d}(\log (2 d / r))^{y_{2}-1}\left(\log \left(2 d^{\prime} / r\right)\right)^{y_{2}-1} y_{2}^{\Omega(r)} / r .
\end{aligned}
$$


Par sommation d'Abel, la dernière somme en $r$ est

$$
\ll \int_{1}^{d}(\log (2 d / r))^{y_{2}-1}\left(\log \left(2 d^{\prime} / r\right)\right)^{y_{2}-1}(\log r)^{y_{2}-1} r^{-1} d r .
$$

Décomposons la dernière intégrale en $I_{1}+I_{2}$ ayant respectivement $[1, \sqrt{d}]$ et $[\sqrt{d}, d]$ pour domaines d'intégration. On a

$$
I_{1} \ll(\log d)^{2 y_{2}-1}\left(\log d^{\prime}\right)^{y_{2}-1} .
$$

Par ailleurs, si $d^{\prime}>d^{2}$ alors $I_{2} \ll\left(\log d^{\prime}\right)^{y_{2}-1}(\log d)^{2 y_{2}-1}$. Dans le cas $d<d^{\prime} \leq d^{2}$ on a

$$
\begin{aligned}
I_{2} & \ll(\log d)^{y_{2}-1} \int_{1}^{d}(\log (2 z))^{y_{2}-1}\left(\log \left(2 z d^{\prime} / d\right)\right)^{y_{2}-1} z^{-1} d z \\
& \ll(\log d)^{y_{2}-1}\left\{\left(\log \left(2 d^{\prime} / d\right)\right)^{2 y_{2}-1}+\int_{d^{\prime} / d}^{d}(\log (2 z))^{2 y_{2}-2} d z\right\} .
\end{aligned}
$$

Donc, si $d<d^{\prime} \leq d^{2}$ et si $y_{2} \leq 1 / 2$ alors

$$
I_{2} \ll(\log d)^{y_{2}-1}\left(\log \left(2 d^{\prime} / d\right)\right)^{2 y_{2}-1} \log _{2} n .
$$

Or, cette majoration est $\gg(\log d)^{2 y_{2}-1}(\log d)^{y_{2}-1} \log _{2} n$ lorsque $d^{\prime}>d^{2}$. Par conséquent, on a dans tous les cas

$$
\begin{aligned}
\sum_{r \leq d}(\log (2 d / r))^{y_{2}-1}\left(\log \left(2 d^{\prime} / r\right)\right)^{y_{2}-1} y_{2}^{\Omega(r)} / r & \\
& \ll(\log d)^{y_{2}-1}\left(\log \left(2 d^{\prime} / d\right)\right)^{2 y_{2}-1} \log _{2} n .
\end{aligned}
$$

D'où

$$
S_{2}\left(X ; n, d, d^{\prime}\right) \ll X(\log n)^{\alpha_{2}}\left(\log _{2} n\right)^{3}(\log d)^{y_{2}-1}\left(\log \left(2 d^{\prime} / d\right)\right)^{2 y_{2}-1}
$$

avec $\alpha_{2}=y_{2}-1-\beta \log y_{2}-2 \lambda$ et puisque $\log d \geq \log P^{+}(d) \geq \log T(n)=$ $(\log n)^{1-\varepsilon}$, on a

$$
S_{2}\left(X ; n, d, d^{\prime}\right) \ll X(\log n)^{\gamma_{2}+o(1)}\left(\log \left(d^{\prime} / d\right)\right)^{2 y_{2}-1}
$$

avec $\gamma_{2}=2 y_{2}-2-\beta \log y_{2}-2 \lambda$.

Par le lemme 7 , si $1-2 y_{2} \leq 0.67$, soit $y_{2} \geq 0.165$, on en déduit

$$
\sum_{\substack{d, d^{\prime} \mid n \\(d), P^{+}\left(d^{\prime}\right)>T(n)}} \mu\left(d d^{\prime}\right)^{2} S_{2}\left(X ; n, d, d^{\prime}\right) \ll X(\log n)^{A+o(1)} \quad \text { p.p. }
$$

avec

$$
A=\gamma_{2}+\log 4+2 y_{2}-1=4 y_{2}-3-\beta \log y_{2}-2 \lambda+\log 4 .
$$


On choisit $y_{2}=\beta / 4 \geq 1 / 4$, de sorte que la condition $y_{2} \geq 0.165$ est bien réalisée. On obtient donc

$$
\sum_{\substack{d, d^{\prime} \mid n \\ P^{+}(d), P^{+}\left(d^{\prime}\right)>T(n)}} \mu\left(d d^{\prime}\right)^{2} S_{2}\left(X ; n, d, d^{\prime}\right) \ll X(\log n)^{-F(\lambda)+o(1)} \text { p.p. }
$$

Nous sommes maintenant en mesure de majorer

$$
\sum_{\substack{d, d^{\prime} \mid n \\ P^{+}(d), P^{+}\left(d^{\prime}\right)>T(n) \\ d^{\prime}>d}} \mu(d)^{2} \mu\left(d^{\prime}\right)^{2} S_{1}\left(X ; n, d, d^{\prime}\right) .
$$

Pour cela, en faisant appel aux lemmes 1 et 3 on a pour $d^{\prime}>d$,

$$
\begin{aligned}
S_{1}\left(X ; n, d, d^{\prime}\right) \ll & X(\log n)^{y_{1}-1-\beta \log y_{1}} \\
& \times \sum_{d<t \leq(1+\eta) d} y_{1}^{\Omega(t)} / t \sum_{d^{\prime} / t<s^{\prime} \leq(1+\eta) d^{\prime} / t} y_{1}^{\Omega\left(s^{\prime}\right)} / s^{\prime} \\
\ll & X(\log n)^{y_{1}-1-\beta \log y_{1}-\lambda}(\log (1 / \eta))^{1-y_{1}} \\
& \times \sum_{d<t \leq(1+\eta) d}\left(\log \left((1+\eta) d^{\prime} / t\right)\right)^{y_{1}-1} y_{1}^{\Omega(t)} / t \\
\ll & X(\log n)^{y_{1}-1-\beta \log y_{1}-\lambda}\left(\lambda \log _{2} n\right)^{1-y_{1}} \\
& \times\left(\log \left(d^{\prime} / d\right)\right)^{y_{1}-1} \sum_{d<t \leq(1+\eta) d} y_{1}^{\Omega(t)} / t \\
\ll & X(\log n)^{y_{1}-1-\beta \log y_{1}-2 \lambda}\left(\log _{2} n\right)^{2-2 y_{2}} \\
& \times\left(\log \left(d^{\prime} / d\right)\right)^{y_{1}-1}(\log d)^{y_{1}-1} .
\end{aligned}
$$

Posant $\alpha_{1}:=2 y_{1}-2-\beta \log y_{1}-2 \lambda$, il suit

$$
\begin{aligned}
& \sum_{\substack{d, d^{\prime} \mid n \\
d^{\prime}>3 d \\
(d)>T(n)}} \mu(d)^{2} \mu\left(d^{\prime}\right)^{2} S_{1}\left(X ; n, d, d^{\prime}\right) \\
& \quad \leq X(\log n)^{\alpha_{1}+\varepsilon} \sum_{\substack{d, d^{\prime} \mid n \\
d^{\prime} \neq d}}\left(1+\left|\log \left(d^{\prime} / d\right)\right|\right)^{y_{1}-1} \mu(d)^{2} \mu\left(d^{\prime}\right)^{2} .
\end{aligned}
$$

On a de même

$$
\sum_{\begin{array}{c}
d, d^{\prime} \mid n \\
d^{\prime}>3 d \\
P^{\prime}(d)>T(n)
\end{array}} \mu(d)^{2} \mu\left(d^{\prime}\right)^{2} S_{1}\left(X ; n, d, d^{\prime}\right) \leq X(\log n)^{-F(\lambda)+o(1)} \quad \text { p.p. }
$$


Par ailleurs, dans le cas $d<d^{\prime} \leq 3 d$ l'inégalité (3.10) implique

$$
S_{1}\left(X ; n, d, d^{\prime}\right) \ll X(\log n)^{y_{1}-1-\beta \log y_{1}} \sum_{d<t \leq(1+\eta) d} y_{1}^{\Omega(t)} / t .
$$

Par le lemme 3 on a donc

$$
S_{1}\left(X ; n, d, d^{\prime}\right) \ll X(\log n)^{2 y_{1}-2-\beta \log y_{1}-\lambda} .
$$

Il en découle

$$
\begin{aligned}
\sum_{\substack{d, d^{\prime} \mid n \\
d<d^{\prime} \leq 3 d \\
P^{+}(d)>T(n)}} S_{1}\left(X ; n, d, d^{\prime}\right) & \ll X(\log n)^{2 y_{1}-2-\beta \log y_{1}-\lambda} 2^{\omega(n)} \\
& \ll X(\log n)^{-\gamma_{1}+\varepsilon} \quad \text { p.p. }
\end{aligned}
$$

où l'on a posé $\gamma_{1}=-2 y_{1}+2+\beta \log y_{1}+\lambda-\log 2$. Le choix $y_{1}=\beta / 2$ implique $\gamma_{1}=F(\lambda)$.

Enfin, il reste à majorer

$$
\sum_{\substack{d \mid n \\ P^{+}(d)>T(n)}} \mu(d)^{2} S_{0}(X ; n, d) .
$$

On a

$$
=\sum_{\substack{m \leq X \\ \Omega(m, 3 n) \leq \beta \log _{2} n}} \sum_{\substack{t \mid m \\ d<t \leq(1+\eta) d}} 1+2 \sum_{\substack{m \leq X \\ \Omega(m, 3 n) \leq \beta \log _{2} n}} \sum_{\substack{t, t^{\prime} \mid m \\ d<t<t^{\prime} \leq(1+\eta) d}} 1 .
$$

D'une part, pour $0<y_{0} \leq 1 / 2$ on a

$$
\sum_{\substack{m \leq X \\ \Omega(m, 3 n) \leq \beta \log _{2} n}} \sum_{\substack{t \mid m \\ d<t \leq(1+\eta) d}} 1 \leq(\log n)^{-\beta \log y_{0}} \sum_{m \leq X} y_{0}^{\Omega(m, 3 n)} \sum_{\substack{t \mid m \\ d<t \leq(1+\eta) d}} 1 .
$$

La dernière somme en $m$ est majorée par

$$
\begin{aligned}
\sum_{d<t \leq(1+\eta) d} y_{0}^{\Omega(t)} \sum_{m \leq X / t} y_{0}^{\Omega(m, 3 n)} & \ll X(\log n)^{y_{0}-1} \sum_{d<t \leq(1+\eta) d} y_{0}^{\Omega(t)} / t \\
& \ll X(\log n)^{y_{0}-1-\lambda}(\log d)^{y_{0}-1}
\end{aligned}
$$

en utilisant le lemme 3. Or,

$$
\sum_{\substack{d \mid n \\ P^{+}(d)>T(n)}}(\log d)^{y_{0}-1} \mu(d)^{2} \leq(\log n)^{y_{0}-1+\log 2+2 \varepsilon} \quad \text { p.p. }
$$


Ainsi, le choix $y_{0}=\beta / 2 \leq 1$ implique

$$
\begin{aligned}
\sum_{\substack{d \mid n \\
P^{+}(d)>T(n)}} \mu(d)^{2} \sum_{\substack{m \leq X \\
\Omega(m, 3 n) \leq \beta \log _{2} n}} \sum_{\substack{t \mid m \\
d<t \leq(1+\eta) d}} 1 \\
\leq X(\log n)^{-F(\lambda)+o(1)} \quad \text { p.p. }
\end{aligned}
$$

Par ailleurs, en majorant la fonction indicatrice de $\Omega(m, 3 n) \leq \beta \log _{2} n$ par $y^{\Omega(m, 3 n)-\beta \log _{2} n}, 0<y \leq 1$, on a d'après le lemme 3 ,

$$
\begin{gathered}
\sum_{\substack{d \mid n \\
P^{+}(d)>T(n)}} \mu(d)^{2} \sum_{\substack{m \leq X \\
\Omega(m, 3 n) \leq \beta \log _{2} n}} \sum_{\substack{t, t^{\prime} \mid m \\
d<t<t^{\prime} \leq(1+\eta) d}} 1 \\
\leq X(\log n)^{-H_{\lambda}+3 \varepsilon} \\
\leq \text { p.p. }
\end{gathered}
$$

où l'on a posé

$$
H_{\lambda}=-4 y+3+2 \lambda-\log 2+\beta \log y .
$$

Le choix $y=\beta / 4$ implique

$$
H_{\lambda}= \begin{cases}Q(\beta)+\log 2 & \text { si } \log 4-1<\lambda<\log 8-1, \\ 2 \lambda+1-\log 8 & \text { si } \lambda \geq \log 8-1\end{cases}
$$

Et puisque $H_{\lambda} \geq F(\lambda)$, on a alors en vertu de (3.20)-(3.22),

$$
\sum_{\substack{d \mid n \\ P^{+}(d)>T(n)}} \mu(d)^{2} S_{0}(X ; n, d) \leq X(\log n)^{-F(\lambda)+o(1)} \quad \text { p.p. }
$$

En conséquence, il découle de (3.9), (3.10), (3.16), (3.19) et (3.23) que

$$
\sum_{m \in \mathcal{S}} \tau\left(m, \mathcal{D}_{n}^{*}\right)^{2} \leq X(\log n)^{-F(\lambda)+o(1)} \quad \text { p.p. }
$$

Ainsi, par (3.7), (3.8) et (3.24) on obtient pour $\lambda>\log 4-1$,

$$
\mathbf{d} \mathcal{B}_{\lambda}(n) \geq(\log n)^{-F(\lambda)+o(1)} \quad \text { p.p. }
$$

4. La majoration de $\mathbf{d} \mathcal{B}_{\lambda}(n)$. Notre point de départ est la majoration

$$
\begin{aligned}
B(X ; n, \lambda) & =\left|\left\{m \leq X: \tau\left(m, \mathcal{D}_{n}\right)>0\right\}\right| \\
& \leq \sum_{\substack{m \leq X \\
\Omega(m, 3 n)>\beta \log _{2} n}} 1+\sum_{m \leq X} y^{\Omega(m, 3 n)-\beta \log _{2} n} \tau\left(m, \mathcal{D}_{n}\right)
\end{aligned}
$$

où $X \geq X_{0}(n), 0<y \leq 1$ et $\lambda \geq \log 4-1$. D'une part, en suivant une démonstration analogue à celle du théorème 010 de [HT88] on a

$$
\sum_{\substack{m \leq X \\ \Omega(m, 3 n) \geq \beta \log _{2} n}} 1 \ll X(\log n)^{-Q(\beta)} ;
$$


d'autre part, en faisant appel aux lemmes 1 et 2 on a

$$
\begin{aligned}
& \sum_{m \leq X} y^{\Omega(m, 3 n)-\beta \log _{2} n} \tau(m, \mathcal{D}) \\
& \leq(\log n)^{-\beta \log y} \sum_{d \mid n} \sum_{d<t \leq(1+\eta) d} y^{\Omega(t)} \sum_{m \leq X / t} y^{\Omega(m)} \\
& \leq X(\log n)^{-\beta \log y+y-1} \sum_{d \mid n} \sum_{d<t \leq(1+\eta) d} y^{\Omega(t)} / t \\
& \ll X(\log n)^{-\beta \log y+y-1-\lambda}(\log (1 / \eta))^{1-y} \sum_{\substack{d \mid n \\
d \neq 1}}(\log d)^{y-1} .
\end{aligned}
$$

Or, on sait que

$$
\sum_{\substack{d \mid n, d \neq 1 \\ P^{+}(d)<\log n}}(\log d)^{y-1} \leq 2^{\Omega(n, \log n)} \leq \log _{2} n \quad \text { p.p. }
$$

Par ailleurs on a

$$
\begin{aligned}
\sum_{\substack{d \mid n \\
P^{+}(d)>\log n}}(\log d)^{y-1} & \ll \sum_{\log _{3} n<k \leq \log _{2} n} e^{(y-1) k} \sum_{\substack{d \mid n \\
k-1<\log _{2} P^{+}(n) \leq k}} 1 \\
& \ll \sum_{\log _{3} n<k \leq \log _{2} n} e^{(y-1) k} 2^{\Omega\left(n, \exp e^{k}\right)} .
\end{aligned}
$$

Utilisant l'inégalité suivante donnée par le lemme 50.1 de [HT88] :

$$
\max _{\log _{3} n<k \leq \log _{2} n} \Omega\left(n, \exp e^{k}\right) / k \leq 1+\left(1 / \log _{4} n\right) \quad \text { p.p. }
$$

nous obtenons ainsi pour $1-\log 2<y \leq 1$,

$$
\begin{aligned}
\sum_{\substack{d \mid n \\
P^{+}(d)>\log n}}(\log d)^{y-1} & \\
& \ll \sum_{k \leq \log _{2} n} \exp \left\{\left(y-1+\log 2+\left(\log _{4} n\right)^{-1}\right) k\right\} \quad \text { p.p. } \\
& \ll(\log n)^{y-1+\log 2+o(1) \quad \text { p.p. }}
\end{aligned}
$$

Par conséquent, nous avons

$$
\begin{aligned}
& B(X ; n, \lambda) \\
& \quad \ll X(\log n)^{-Q(\beta)}+X(\log n)^{2 y-2+\log 2-\beta \log y-\lambda+o(1)} \quad \text { p.p. }
\end{aligned}
$$


Dans le cas $\log 4-1 \leq \lambda \leq 3 \log 2-1$ nous obtenons donc en choisissant $y=B / 2 \geq \log 2>1-\log 2$,

$$
B(X ; n, \lambda) \leq X(\log n)^{-Q(\beta)+o(1)} \quad \text { p.p. }
$$

Par ailleurs, si $\lambda>3 \log 2-1$ on a

$$
\begin{aligned}
B(X ; n, \lambda) & \leq X \sum_{d \mid n} \sum_{d<t \leq(1+\eta) d} 1 / t \ll X(\log n)^{-\lambda} 2^{\Omega(n)} \\
& \leq X(\log n)^{\log 2-\lambda+o(1) \quad \text { p.p. }}
\end{aligned}
$$

En conséquence, pour $\lambda>\log 4-1$ on a

$$
B(X ; n, \lambda) \leq X(\log n)^{-F(\lambda)+o(1)} \quad \text { p.p. } \quad\left(X \geq X_{0}(n)\right) .
$$

\section{Bibliographie}

[B48] F. A. Behrend, Generalization of an inequality of Heilbronn and Rohrbach, Bull. Amer. Math. Soc. 54 (1948), 681-684.

[HR79] H. Halberstam and H.-E. Richert, On a result of R. R. Hall, J. Number Theory 11 (1979), 76-89.

[HR66] H. Halberstam and K. F. Roth, Sequences, Oxford University Press, 1966.

[HT88] R. R. Hall and G. Tenenbaum, Divisors, Cambridge University Press, 1988.

[R93] A. Raouj, Sur la densité de certains ensembles de multiples, 1, ce volume, $121-152$.

[S80] P. Shiu, A Brun-Titchmarsh theorem for multiplicative functions, J. Reine Angew. Math. 313 (1980), 161-170.

[T90] G. Tenenbaum, Introduction à la théorie analytique et probabiliste des nombres, Publications de l'Institut Elie Cartan, Université de Nancy I, 1990.

UNIVERSITÉ CADI AYYAD

FACULTÉ DES SCIENCES, SEMLALIA

DÉPARTEMENT DE MATHÉMATIQUES

B.P.S. 15, MARRAKECH, MAROC 